\title{
Monopile foundation under lateral cyclic action. Numerical modelling
}

\author{
Andrei Valentin Drăgușin ${ }^{1,{ }^{*}}$ and Loretta Batali $^{1}$ \\ ${ }^{1}$ Technical University of Civil Engineering, Geotechnical Department, Bucharest, Romania
}

\begin{abstract}
Foundation of an off-shore wind mill is submitted throughout its existence to a very high number of cycles coming from lateral actions such as waves or wind. These actions have a strong aleatory character which makes them very hard to predict, quantify and analyse. Therefore, in current design practice, these actions are being considered as pseudo-static force at their maximum values, with the cyclic phenomenon being neglected. This can lead to an inappropriate design of the foundation, which could have a negative impact on the future structure. This type of structure is generally built on a monopile foundation, a single, large diameter pile, which will be submitted to thousands lateral cycles. The pile diameter plays an important role, influencing the behaviour of the entire structure. Centrifuge experiments on small-scale models are very useful to study such complex problem as piles under lateral cyclic loads. Several researches have been carried out internationally and the results can be used for calibrating numerical models, which is obviously a more accessible method of design, compared to an experimental approach. This has been precisely the starting point of this paper. The purpose of the present paper is to analyse the influence of the pile diameter, by using a FEM a numerical model, previously calibrated based on centrifuge experiments carried out at IFSTTAR Nantes. For the numerical modelling the software CESAR-LCPC 3D has been used. Several pile diameters have been considered, as follows: $0.72 \mathrm{~m}, 1.08 \mathrm{~m}, 1.44 \mathrm{~m}, 1.80 \mathrm{~m}, 2.16 \mathrm{~m}$ and $2.52 \mathrm{~m}$. The results are taking into account the lateral displacement and bending moment of the piles, for static and cyclic loading. The main objective was to determine the stabilisation rate of the most important two design elements (pile head displacement and maximum bending moment) after " $n$ " cycles and to eventually conclude the diameter value beyond which no more influence of cycles is recorded. The numerical model considered 15 cycles and the results have been used extrapolated in order to determine the cycle „n” of stabilisation (for displacement and bending moment).
\end{abstract}

\section{Introduction}

Current design practice of piles submitted to lateral cyclic actions coming from waves or wind is often ignoring the cyclic effect of the loading due to the complexity of the soil-structure interaction involved. Numerical models are widely used nowadays, but they have to be calibrated based on experimental results to be further used in design practice. The paper is part of a broader study aiming at analysing the main parameters of influence for this case. Given the fact that most of the time offshore windmills are founded on a monopile, this paper analyses the influence of the pile diameter on its cyclic behaviour.

In order to study the influence of a single pile submitted to lateral cyclic action in sandy soil, a numerical model has been developed, using the 3D finite element software CESAR-LCPC, which has been previously calibrated based on centrifuge tests on smallscale pile model. The numerical model was now used for studying the influence of the pile diameter.
Six piles having different diameter were considered, the rest of the characteristics remaining the same. All of the piles are fully circular steel ones, with the length beneath the soil surface of $12 \mathrm{~m}$ and above soil surface of $1.6 \mathrm{~m}$. The piles diameters are presented in the table 1 below.

Table 1. Main pile characteristics.

\begin{tabular}{|c|c|c|c|c|c|}
\hline Pile 1 & Pile 2 & Pile 3 & Pile 4 & Pile 5 & Pile 6 \\
\hline B & B & B & B & B & B \\
$0.72 \mathrm{~m}$ & $1.08 \mathrm{~m}$ & $1.44 \mathrm{~m}$ & $1.80 \mathrm{~m}$ & $2.16 \mathrm{~m}$ & $2.52 \mathrm{~m}$ \\
\hline
\end{tabular}

The considered soil was a medium to loose Fontainebleau sand, with a unit weight of $15.1 \mathrm{kN} / \mathrm{m}^{3}$, a deformation modulus of $30 \mathrm{MPa}$ and an internal friction angle of $30^{\circ}$, frequently used in France for experiments.

All the piles were submitted to a $960 \mathrm{kN}$ lateral force applied on its head. The cyclic amplitude of loadingunloading was of $480 \mathrm{kN}$, value which is considered to be the most reliable one for a realistic simulation. It should be mentioned that the numerical results obtained

\footnotetext{
* Corresponding author: dragusin andrei_valentin@yahoo.com
} 
for pile 1 in the previously given conditions (soil and loading type), have been initially calibrated against some small-scale experimental data, obtained in centrifuge by [1] and made available to us by IFSTTAR, Nantes. The numerical model and its calibration were previously published in paper [2]. It should also be stated that pile 1 is a flexible one, whereas the five others are rigid. This classification has been based on Ménard's criterion [3], which introduces a transfer length. Obviously, flexible or rigid, piles exhibit different behaviour under lateral cyclic action. This aspect is not approached in the present paper, being published in paper [4].

\section{Numerical models}

The size of the model was $16 \times 8 \times 16 \mathrm{~m}$ in size (xyz), with a mesh density of $2.4 \mathrm{~m}$. For the pile and pile-soil contact the mesh density was $0.4 \mathrm{~m}$. A tetrahedron mesh TETGEN with a cubic interpolation mesh creating function was used. Thus, it resulted six models with an approximate number of nodes comprised between 30000 and 40000 (the smallest for pile 1, the largest for pile 6). All piles were considered as being free on head. Figure 1 below shows the mesh and the boundary limits for the pile 1 .

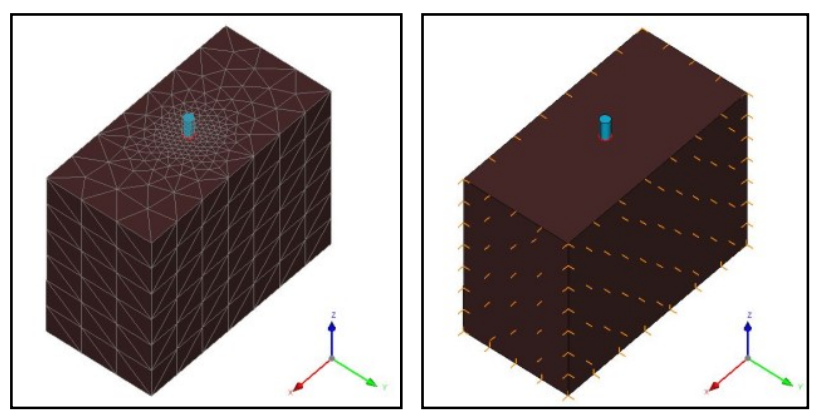

Fig. 1. Mesh and boundary limits of the model-CESAR-LCPC.

In first phase, the piles were submitted to a lateral monotonic loading in six increments until reaching the maximum value of $960 \mathrm{kN}$. After that, 30 cycles of 480 $\mathrm{kN}$ amplitude were applied, each in only 3 increments, due to a long calculation time.

The pile-soil interface was considered as being perfectly sliding and having a nil traction strength.

As for the constitutive law of the soil, DrückerPrager's plasticity criterion combined with Chaboche's [5] kinematic hardening law (already implemented in CESAR-LCPC 3D software) was considered. The kinematic law and its use in the numerical model are presented in paper [6].

\section{Results for 15 cycles}

Here below are presented the results obtained after using the numerical mode, for 15 cycles.

\subsection{Displacement accumulation on pile head}

Figure 2 presents the rate of lateral displacement accumulation on pile head along the cycles and one can easily observe that the pile diameter plays a major role in the behaviour under lateral cyclic actions.

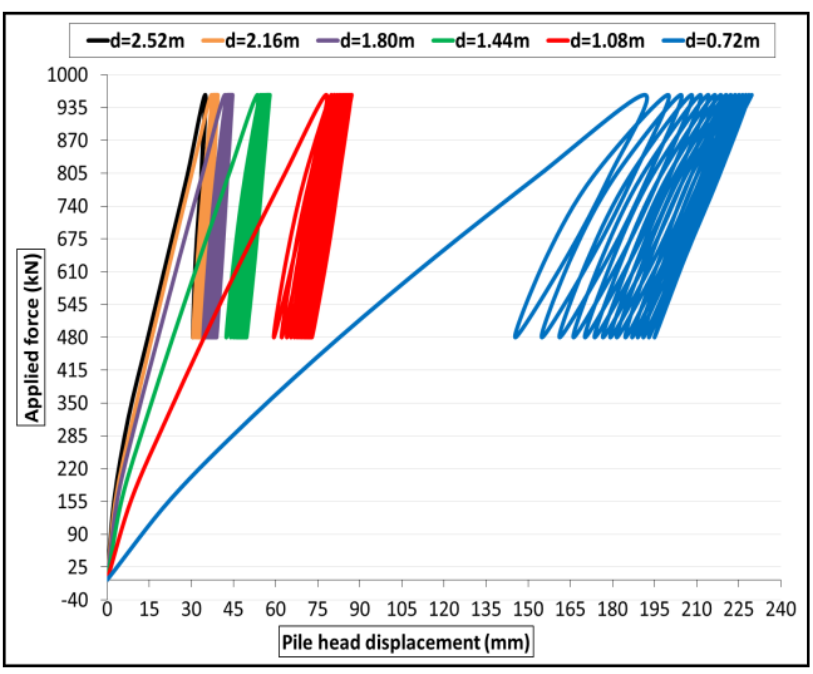

Fig. 2. Displacement accumulation rate on pile head along cycles.

There is an important difference regarding the maximum pile head displacement between pile $1(0.72 \mathrm{~m}$ diameter) and pile 2 (1.44 $\mathrm{m}$ diameter), obviously coming from the difference in stiffness. This difference becomes less important when diameter increases. The stabilisation rate of the pile head displacement is obtained faster for the piles with a large diameter versus the smaller ones, which is emphasized on the graph figure 2 by the steeper slope obtained for large diameters. The hysteretic loops are also smaller for large diameters, which indicates again a faster stabilisation.

Maybe the most interesting result is for pile 6 (2.52 $\mathrm{m}$ diameter), for which only a single hysteretic loop has been obtained, meaning that no matter the phase of loading-unloading the path is the same indefinitely, so the cycles don't have any effect on this pile.

Therefore, pile 6 can be further designed as a pile submitted to a simple monotonic load. This fact has also been confirmed by the pile deformation and by the bending moment diagram (figures 4 and 5). However, for the other 5 piles having smaller diameters, the lateral displacement is increasing along the cycles.

The accumulation rate shown on the graph above (figure 2) wouldn't have been possible to obtain without the implemented kinematic hardening law.

\subsection{Evolution of pile head displacement}

Figure 3 describes the maximum pile head displacement along the 15 considered cycles.

It can be observed a less steep slope with the diameter increase, until reaching stabilisation for pile 6 (2.52 m diameter). line.

This stabilisation is illustrated by the final horizontal

Intentionally, the flexible pile $1(0.72 \mathrm{~m}$ diameter $)$ was not included in the graph figure 3 due to high differences against the other piles. 
The graph in that case wouldn't have been as relevant as it is, the slope being very hard to observe, due to scale problems.

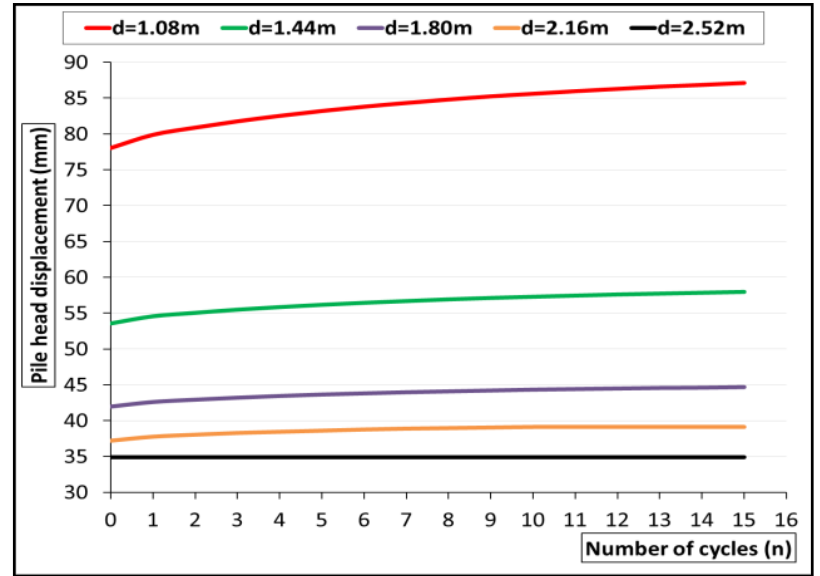

Fig. 3. Pile head displacement evolution along cycles.

\subsection{Pile deformation}

The graph in figure 4, showing the pile deformation vs. depth, is in good agreement with the general behaviour of flexible vs. rigid piles. Pile 1, which is flexible, has the highest deformation and it can fail due to high bending moment which can overcome the pile resistance, whereas the other piles (2 to 6), being rigid, are deforming less and differently, and failure can be reached due to high rotation at a certain depth, occurring when soil resistance has been exceeded.

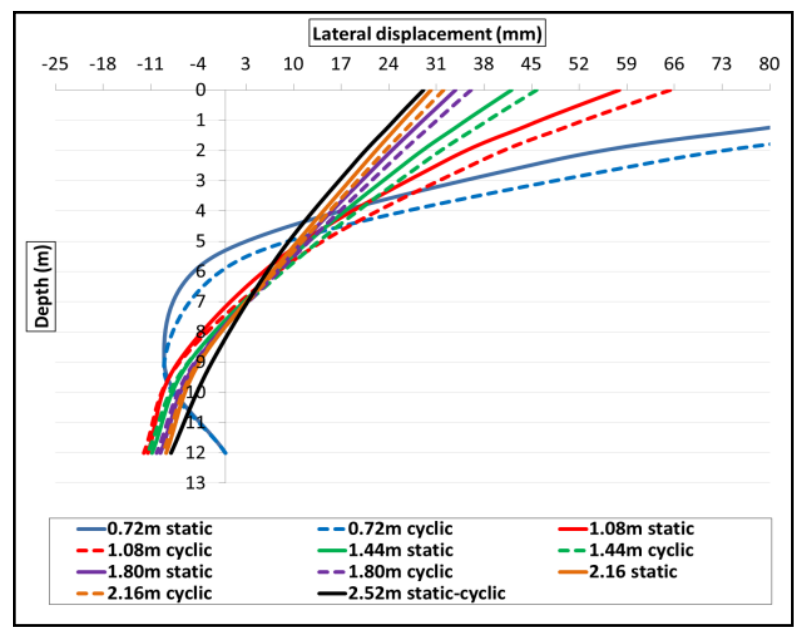

Fig. 4. Pile deformation vs. depth.

It is also interesting to observe that differences between the lateral displacements at the end of the monotonic loading (continuous curve on the graph) and the ones obtained at the end of the $15^{\text {th }}$ cycle (dotted line on the graph) are becoming smaller when diameter is increasing, for pile 6 , both curves being identical. So, even at high depth, the largest pile (no. 6, $2.52 \mathrm{~m}$ diameter) is not influenced by the cyclic effect of loading-unloading.

\subsection{Bending moment diagram}

The next figure (figure 5) shows the bending moment diagram obtained for the 6 piles. It can be easily seen that the cycles are increasing the maximum bending moment developed in the piles. However, for the pile 6, cycles are showing no sign of influence, the bending moment at the end of the monotonic loading being the same as the one calculated at the end of the $15^{\text {th }}$ cycle.

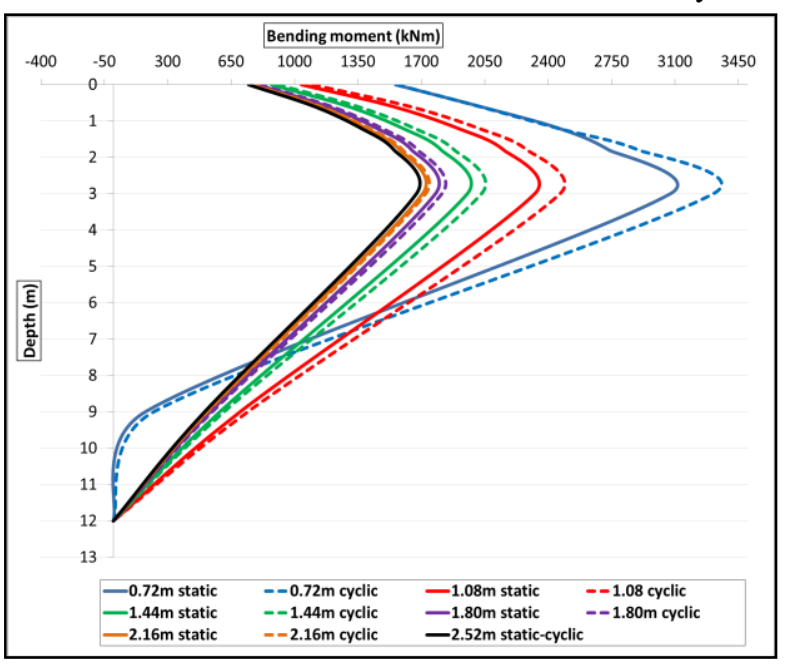

Fig. 5. Bending moment diagram.

On the other hand, there is a slight difference between the diagram shape for pile 1 (flexible) against the others (rigid), considering the fact that mathematically, the moment represents the double derivate of the displacement. The bending moment diagram is in good agreement with the pile deformation (figure 4). However, the maximum bending moment appears in all the piles at about $2.5 \mathrm{~m}$ depth.

\subsection{Maximum bending moment evolution}

The maximum bending moment evolution along cycles (in figure 6) is in a good agreement with the previously presented graphics.

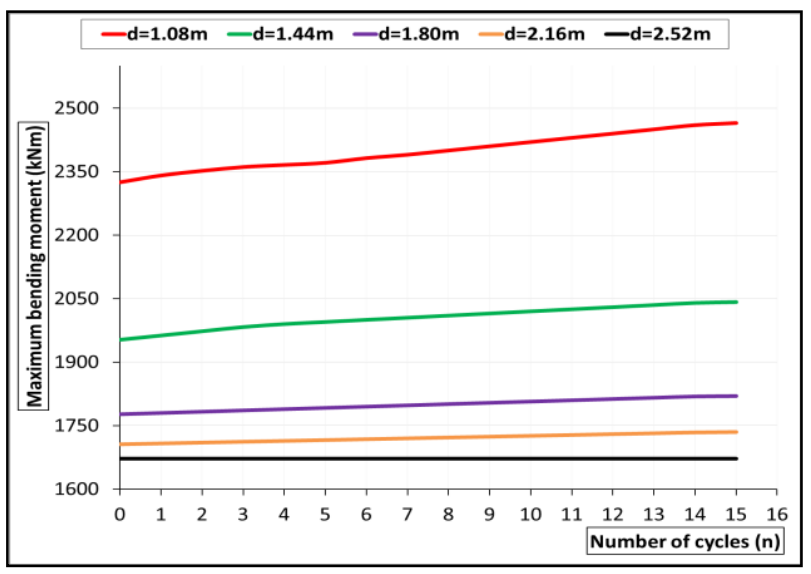

Fig. 6. Maximum bending moment evolution along cycles.

The slope of the graph is steeper for larger diameters, until reaching stabilisation for pile 6 . 


\section{Extrapolation for high number of cycles}

Given the fact that the monopile foundation of an offshore windmill is submitted throughout its existence to a millions cycles of loading-unloading from waves or wind, it was necessary to create some extrapolation functions. The previously 15 analysed cycles in CESARLCPC software served to create these functions. The extrapolation was performed using the non-linear sigmoidal model of Morgan-Mercer-Flodin.

The results are presented below as a synoptic table (table no. 2), with the mention that the calculations are approximate - the obtained values after the cycle " $n$ " of stabilisation have been considered as an integer number.

Table 2. Synoptic table - obtained results after cycle „n” of stabilisation.

\begin{tabular}{|c|c|c|c|c|c|c|c|}
\hline $\begin{array}{c}\text { Pile } \\
\text { diameter } \\
(\mathbf{m})\end{array}$ & A & B & C & D & E & F & G \\
\hline $1-0.72$ & 100000 & 192 & 268 & 40 & 3074 & 3364 & 9 \\
\hline $2-1.08$ & 10000 & 78 & 97 & 25 & 2325 & 2475 & 7 \\
\hline $3-1.44$ & 1000 & 54 & 63 & 17 & 1953 & 2042 & 5 \\
\hline $4-1.80$ & 100 & 42 & 47 & 11 & 1777 & 1833 & 3 \\
\hline $5-2.16$ & 10 & 37 & 39 & 6 & 1706 & 1736 & 2 \\
\hline $6-2.52$ & 1 & 35 & 35 & 0 & 1735 & 1735 & 0 \\
\hline
\end{tabular}

The significance of columns A - G in table 2 is:

A - cycle of stabilisation for pile head displacement and maximum bending moment;

$\mathrm{B}$ - maximum pile head displacement at the end of the monotonic loading $(\mathrm{mm})$;

$\mathrm{C}$ - maximum pile head displacement at the end of the approximate cycle of stabilisation $(\mathrm{mm})$;

$\mathrm{D}$ - increasing of pile head displacement from static to cyclic (\%);

$\mathrm{E}$ - maximum bending moment at the end of the monotonic loading $(\mathrm{kNm})$;

$\mathrm{F}$ - maximum bending moment at the end of the approximate cycle of stabilisation $(\mathrm{kNm})$;

$\mathrm{G}$ - increasing of maximum bending moment from static to cyclic (\%).

As it can be noted, the pile head lateral displacement for pile 1 has a very high increasing from static to stabilisation cycle, of $40 \%$. This percentage is decreasing for larger diameters, until becoming nil for the largest studied diameter $(2.52 \mathrm{~m})$. On the other hand, the increasing of the maximum bending moment is lower, but still having to be considered in the design, especially for small diameter piles.

\section{Conclusions}

Using a previously calibrated numerical model based on small-scale centrifuge tests and developed using CESAR-LCPC 3D software, the paper analyses the influence of the diameter of a monopile submitted to lateral cyclic actions, coming from waves or wind, in sandy soil.
Six piles with same properties, but different diameter have been analysed. The smallest pile was a flexible one, whereas the others were rigid.

The obtained results showed that the increase in the displacement of pile head was of $0-40 \%$ (from the largest to smallest pile), while the increase of the bending moment varied from $0 \%$ to $9 \%$ (also from the larger to the smallest pile), all values compared to monotonic ones. The increase becomes less important for larger piles, while no influence at all was observed for a $2.52 \mathrm{~m}$ diameter pile. This latest, being very rigid, can be simply calculated in the static field with no influence coming from the cycles.

However, this result has been obtained based on a limited number of models, being thus only qualitative.

On the other hand, the results obtained for the other piles with smaller diameters are in a good agreement with other results found in the international literature for sandy soil (pile head displacement accumulation, increasing of lateral pile displacement and maximum bending moment along cycles), as for example [1], [7].

Authors would like to acknowledge dr. David Remaud for providing the licence for CESAR-LCPC 3D software.

\section{References}

1. F. Rosquoët, Pieux sous charge latérale cyclique (PhD thesis, Nantes University, 2004)

2. L. Batali, A. Drăgușin, H. Popa, Numerical modelling by finite elements for a pile foundation under lateral cyclic action (Proceedings of XVI Danube - European Conference on Geotechnical Engineering, Skopje, June 2018)

3. L. Ménard, G. Bourdon, M. Gambin, Méthode générale de calcul d'un pieu sollicité horizontalement en fonction des résultats pressiométriques (Sols-Soils, vol. 22-23, pp. 16-29, 1969)

4. L. Batali, A. Drăgușin, H. Popa, Numerical analysis of the behaviour of a flexible pile foundation versus a rigid one under lateral cyclic action (International Multidisciplinary Scientific GeoConference SGEM, Albena, July 2018) - to be published

5. J. L. Chaboche, S. Forest, J. Besson, G. Cailletaud, Lois de comportement non linéaire des matériaux, 2001

6. A. Drăgușin, Numerical modelling by finite elements for a pile foundation under lateral cyclic action in sandy soil - parameters influence $\left(26^{\text {th }}\right.$ European Young Geotechnical Engineers Conference, ISSMGE, Graz, September 2018) - to be published

7. M. H. J. Rakotonindriana, Comportement des pieux et des groups de pieux sous chargement latéral cyclique ( $\mathrm{PhD}$ thesis, Ecole Nationale des Ponts et Chaussées, Paris, 2009) 